\title{
Additive Construction using Basalt Regolith Fines
}

\author{
Robert P. Mueller ${ }^{1}$, Laurent Sibille ${ }^{2}$, Paul E. Hintze ${ }^{3}$, Thomas C. Lippitt ${ }^{4}$, James G. Mantovani ${ }^{5}$, \\ Matthew W. Nugent ${ }^{6}$, and Ivan I. Townsend ${ }^{7}$ \\ 1,4,5 NASA, Surface Systems Office, Kennedy Space Center, FL 32899 \\ 2 EASI (ESC), Applied Sciences and Technology, Kennedy Space Center, FL 32899 \\ ${ }^{3}$ NASA, Materials Science Division, Kennedy Space Center, FL 32899 \\ ${ }^{6}$ Sierra-Lobo (ESC), Applied Sciences and Technology, Kennedy Space Center, FL 32899 \\ ${ }^{7}$ Craig Technologies (ESC), Applied Sciences \& Technology, Kennedy Space Center, FL 32899
}

\begin{abstract}
Planetary surfaces are often covered in regolith (crushed rock), whose geologic origin is largely basalt. The lunar surface is made of small-particulate regolith and areas of boulders located in the vicinity of craters. Regolith composition also varies with location, reflecting the local bedrock geology and the nature and efficiency of the micrometeorite-impact processes. In the lowland mare areas (suitable for habitation), the regolith is composed of small granules (20 - 100 microns average size) of mare basalt and volcanic glass. Impacting micrometeorites may cause local melting, and the formation of larger glassy particles, and this regolith may contain $10-80 \%$ glass.

Studies of lunar regolith are traditionally conducted with lunar regolith simulant (reconstructed soil with compositions patterned after the lunar samples returned by Apollo). The NASA Kennedy Space Center (KSC) Granular Mechanics \& Regolith Operations (GMRO) lab has identified a low fidelity but economical geo-technical simulant designated as Black Point-1 (BP-1). It was found at the site of the Arizona Desert Research and Technology Studies (RATS) analog field test site at the Black Point lava flow in adjacent basalt quarry spoil mounds.

This paper summarizes activities at KSC regarding the utilization of BP-1 basalt regolith and comparative work with lunar basalt simulant JSC-1A as a building material for robotic additive construction of large structures. In an effort to reduce the import or in-situ fabrication of binder additives, we focused this work on in-situ processing of regolith for construction in a single-step process after its excavation. High-temperature melting of regolith involves techniques used in glassmaking and casting (with melts of lower density and higher viscosity than those of metals), producing basaltic glass with high durability and low abrasive wear. Most Lunar simulants melt at temperatures above $1100^{\circ} \mathrm{C}$, although melt processing of terrestrial regolith at $1500^{\circ} \mathrm{C}$ is not uncommon. These temperatures are achievable by laser heating or by using solar concentrators. Similar to volcanic magma, the cooling rate determines the crystallite size slower cooling develops larger crystals, and rapid quenching can result in fully amorphous glass.
\end{abstract}

\section{INTRODUCTION}

Additive manufacturing is the process of forming an object by sequentially adding and bonding material under computer control, without any waste. It is the opposite of subtractive manufacturing that starts with a larger piece of material and then removes material by methods 
such as cutting, milling, water jetting, and others to create the final desired net shape. Subtractive manufacturing generates substantial waste of both material and energy and restricted tool bit access can prevent the creation of complex shapes, thus forcing complicated designs and assemblies.

Additive Manufacturing is also known as three-dimensional (3D) printing. 3D printers use computer-created digital models to create real-world objects. The printers follow the shape of the model by stacking layer upon layer of material to make the objects. Complex and hollow shapes are possible, resulting in new possibilities for engineering design. In 2013, President Obama stated in his State of the Union address that 3D Printing may revolutionize the way we make almost everything, and that the future of manufacturing is 3D printing. Many existing companies and many new companies are now making 3D printers and advanced print media technology. It is widely believed that $3 \mathrm{D}$ Printing lead to a new $21^{\text {st }}$ century industrial revolution that could substantially impact the manufacturing and building construction markets.

For the purposes of this paper, 3D Additive Manufacturing is sub-divided into two further classifications:

1. 3D Additive Fabrication

2. 3D Additive Construction

The first classification: 3D Additive Fabrication refers to high precision parts (minimum tolerances of $.025 \mathrm{~mm}$ or tighter) that are relatively small in size $\left(<1 \mathrm{~m}^{3}\right)$, and are commonly made of metallic or polymer feedstock. This is common in today's terrestrial industry and most efforts are being focused on advancing the technology in this domain.

The second classification: 3D Additive Construction refers to low tolerance structures that are relatively large $\left(>1 \mathrm{~m}^{3}\right)$, and have low accuracy and precision with manufacturing tolerances on the order of 3 to $6 \mathrm{~mm}$.

This paper focuses on 3D Additive Construction using local resources in outer space in the form of regolith - the loosely consolidated layer of crushed rock and other materials covering the surface of planetary bodies. This could enable construction at distant locations in our solar system (Moon, Mars, Asteroids, outer planets and their moons) without transporting the construction materials through Earth's deep gravity well, with an expensive rocket launch. 3D Additive Construction could provide the solution for extra-terrestrial shelter (electromagnetic space radiation, thermal, micro-meteorites, dust storms, vacuum, fission power plant shielding, rocket blast ejecta at launch/landing, etc.) for human crews and robotic equipment on planetary surfaces. New possibilities for space exploration and space mission architectures may arise out of this technology that is currently under development at the NASA Kennedy Space Center “Swamp Works” innovation labs.

\section{STATE OF THE ART OF 3D ADDITIVE CONSTRUCTION}

Methods for stabilizing lunar regolith and fabricating large structures have been explored since the 1970's. The concepts generally fall in the following categories: mechanical stabilization without additives (compaction, enclosing or "sand bagging"), thermal processing without additives (sintering, melting, glass pulling), stabilization by addition of binders (organic resins, sulfur), and stabilization by chemical reactions (propagating combustion fronts). 
In the past decade, the concept of 3D additive fabrication has permeated building architecture and construction engineering and some technologies have rapidly advanced to demonstrate new construction at full scale in the near future. In 2006, USC Professor Behrokh Khoshnevis proposed developed the "Contour Crafting" concept and has since developed the 3D robotic concrete printer that extrudes a special concrete paste to print houses within 24 hours. Since 2011, Khoshnevis' team have also developed a space version of Contour Crafting to build structures on the lunar and Martian surface using sulfur found in the regolith as a binder for the extruded mixture (Khoshnevis, 2013). This elegant concept offers some powerful advantages for space applications. The additive construction is achieved in a freestanding mode without the need to lay down a bed of supporting feedstock material and thus eliminates the need to excavate such a large volume to uncover the finish structure. This also ensures that the energy expanded in the process is efficiently applied to the construction of the finished product and no waste energy is spent on pre- and post-construction site preparation and excavation.

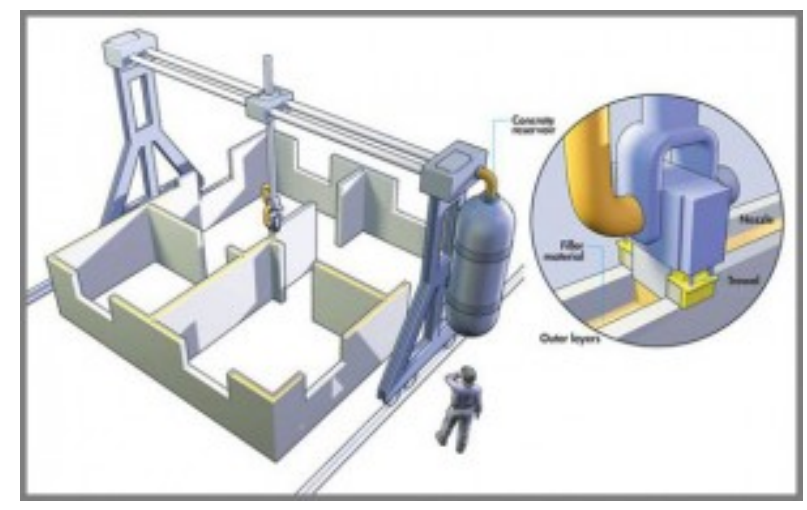

Figure 1. Illustration of Contour Crafting concept for 3D additive construction of homes (courtesy USC/B. Khoshnevis, www.contourcrafting.org)

In 2014, the creation of 3D printed functional buildings was reported; the company Yingchuang New Materials Inc. was the first to build ten 1-room structures in one day with four 3D printers using fused deposition modeling (FDM) technology (Mearian, 2014). The firm had previously constructed its own 33,000 sq. ft. factory in one month using the process.

Enrico Dini of Monolite UK Ltd. has created large intricate walls and closed sculptures to demonstrate a layering process that deposits a mixture of fine sand and metallic oxide (MgO) that undergoes cross-linking when sprayed with a chlorine-based saltwater solution to produce a material similar in properties to sandstone or limestone. The buildup is done on a bed of material that the machine lays down at each pass that supports the erected structure during cross-linking and consolidation. Consequently, it requires a lot of excavating to free up the finished structure. In 2013, the European Space Agency (ESA) selected Dini's D-Shape process to develop the concept of additive construction of a lunar base using a mixture of lunar regolith basaltic simulant and MgO. The project created a 1.5-ton monolithic structure at construction rate of 2 $\mathrm{m} / \mathrm{s}$ and has begun testing under vacuum.

Recently, Howe et al. introduced a concept to equip the multi-functional robotic platform AllTerrain Hex-Limbed Extra-Terrestrial Explorer (ATHLETE) with an end-effector that could interface with several print head designs and construction processes (Howe, 2013). The proposed 
approach is focused on using printing concepts capable of creating freestanding structures that include the proven method of Contour Crafting. It also envisions the use of urethane binder/regolith mixtures demonstrated by Adherent Technologies (Gosau, 2012) and solar concentrators demonstrated by Physical Sciences, Inc. (Nakamura, 2011). Howe and coworkers also have proposed a print head concept based on the integrated use of a resonant cavity for microwave processing of a regolith flow.

Recent collaborative work performed by NASA KSC and Washington State University. focused on fabricating small cylindrical test articles (8-10 mm diam., height $25-30 \mathrm{~mm}$ ) by direct melting of lunar basalt simulant JSC-1AC, established feasibility of the processing approach. The samples created with a Nd-YAG laser set at $50 \mathrm{~W}$ with translational speed of $20 \mathrm{~mm} / \mathrm{s}$ and a regolith feed rate of $\sim 12.3 \mathrm{~g} / \mathrm{min}$ displayed a mostly amorphous microstructure obtained after passive cooling. While mechanical properties were not reported for the resulting defect-free parts, the authors indicated that such parts could be strong enough to make nuts and bolts.

Our team reviewed these accomplishments and performed a trade study of a wide range of existing and novel concepts and supporting technologies. Our approach focused on defining a process that would be applicable to space environments and involve little or no imported materials from Earth. Several major issues were found to be prominent in their impact on the trade study as follows:

- Despite the known presence of water on Earth's moon and Mars, our knowledge of the resources (quantities, spatial distribution, characteristics) on both planetary bodies is still very uncertain at this time. In addition, the ground confirmation of large quantities of water in lunar and Martian subsurface will likely lead to its use (after energy-intensive extraction from the ground) as a source of oxygen and hydrogen to produce propellants and life support consumables, before being consumed in the manufacturing of concrete.

- The extraction of other in-situ binders on extraterrestrial bodies is challenging but may be a viable option if such resources are in concentrated ore or deposits. If the compounds being sought are too scarce in the regolith, such operation will increase the excavated mass by orders of magnitude thus leading to larger hardware mass, engineering complexity and multiply the failure risks for the whole process. In concentrated form, the binder may need to be pre-sorted and quantified before being remixed with regolith to obtain consistent finished products.

- Excavation of the various regoliths in planetary environments characterized for their vacuum conditions, very high temperature gradients, and significant electrostatic charging is a major engineering challenge. In terrestrial conditions, soil handling equipment display some of the highest failure rates and maintenance costs per operational hour among industrial processes in spite of a long history of practice and knowledge of soils. It is critical to use excavated regolith with maximum efficiency in the processed construction products.

- The project undertaken by the Swamp Works team received a finite amount of funding to establish that structural elements could be created repeatedly by 3D additive construction of low complexity and did not allow the extensive development of any technology for which the materials processing component is unknown or largely untested such as a new binder. 
Our examination of the state-of-the-art of applicable technologies and the above considerations led us to prioritize the adoption of a process that would erect freestanding structures with direct energy input to the regolith during material deposition and the avoidance of additives or binders in the first phase of our project. Sintering and melting of regolith emerged as the process of choice with a variety of energy sources being considered among which radiated heating (microwave, laser or Infrared) was favored.

\section{KSC ACTIVITIES USING BASALT REGOLITH FOR ROBOTIC ADDITIVE CONSTRUCTION OF LARGE STRUCTURES ON PLANETARY SURFACES}

It is a goal of the Surface Systems group at KSC to investigate novel in-situ construction (ISC) technologies that rely solely on the use of local regolith to fabricate parts and structures on the surfaces of Asteroids, the Moon, Mars and other planetary bodies. If successful, this approach to construction on planetary surfaces will be a game-changing technology that results in higher reliability and safety for surface operations. It will also lead to the development of a robust architecture for space mission logistics that enables the expansion of human and robotic exploration into the solar system.

Recent work in the NASA Kennedy Space Center's Surface Systems group and at the University of Southern California (USC) under two NASA Innovative Advanced Concepts (NIAC) awards have demonstrated promising results by utilizing planetary regolith simulant materials which could fabricate heat shields, bricks, landing/launch pads, berms, roads, and other structures that could be fabricated using regolith that is sintered by itself or mixed with a polymer binder. The technical goals and objectives of current research efforts at KSC are to prove the feasibility of 3D printing by additive construction using planetary regolith and to show that the resulting materials and products have structural integrity and practical applications in space exploration.

KSC has gained knowledge in this area through in house work and several NASA Small Business Innovative Research (SBIR) contracts (Ceralink, Adherent, Honeybee, Orbitec), collaborations with universities (USC NIAC project: "ISRU-Based Robotic Construction Technologies for Lunar and Martian Infrastructures”), as well as NASA collaborative research (KSC NIAC project: "Regolith Derived Heat Shield," which produced test coupons that were tested at the Arc Jet Test Facility located at NASA Ames Research Center). These previous results indicate that the unique physical properties of granular planetary regolith are well suited for use as a construction material with high thermal insulation properties, low densities and good manipulation characteristics. Examples of regolith manipulation processes are solar heat sintering, microwave sintering, polymer binders, compaction, regolith paste extrusion and lunacrete forming.

Methods of transferring regolith to a 3D print head mounted on a robotic arm are being developed at KSC to investigate the feasibility of adhering the regolith particles together in successive 2D layers to achieve a 3D printing proof-of-concept process based on additive manufacturing. This will allow us to fabricate useful structures such as blast walls, landing pads, habitats, bricks, roads, antenna towers, heat shields and even propellant tanks. A functioning 3D regolith printer will result in substantial mass savings by using local regolith without requiring additional material and equipment to be transported from Earth. The logistics required to set up a human outpost on another planetary surface are vast and prohibitively expensive. Space transportation costs are currently high, ranging from $\$ 4,000$ to $\$ 13,000$ per $\mathrm{kg}$, (or more) when launched from the ground to Low Earth Orbit (LEO). Thus the potential savings value of ISC to 
fabricate structures and spare parts in-situ using local materials on a planetary surface is also high.

The Surface Systems group at KSC has decided to focus on additive construction methods that only utilize local regolith materials as feedstock. Alternative methods that are not being studied at KSC are those that require importing non-native materials. For example, regolith can be stabilized by mixing with granular minerals with polymers, clays, sulfur and low melting point metals. Certain metal powders, such as aluminum and magnesium, can be used as another type of imported binder material for self-sintering processes in which the metal powder chemically reacts with mineral oxides at a sufficiently high temperature produced by an electrical ignition. Such binder materials are not likely to easily or reliably obtained by processing local regolith. On the other hand, some planetary environments possess significant deposits of solid volatiles, such as water ice and carbon dioxide ice, which can be utilized as binder materials to stabilize regolith if only temporarily.

The approach that KSC has chosen to pursue is in the area of additive construction by either directly melting or sintering regolith materials to print 3D structures. A number of techniques were considered for heating regolith in terrestrial and planetary environments, including resistive heating, microwave heating, focused solar heating, and laser heating. After conducting a trade study of the possible methods of developing a 3D regolith printer, KSC selected laser heating using an infrared laser as the most direct method to deliver a sufficient amount of energy into a small volume that will allow granular regolith particles to fuse together. The laser also allows for either the sintering or melting of regolith depending on the desired final state of the fused regolith product. It is not yet known whether or not laser sintering or laser melting of regolith will ultimately prove to be feasible for use on a planetary surface due to power constraints. However, by combining a laser with a regolith feed system in a print-head that is robotically controlled, a terrestrial 3d regolith printer can yield a variety of structures that can be studied for their physical and mechanical properties.

KSC has installed a large industrial FANUC Model M-410iC robotic arm in the "Swamp Works" Granular Mechanics and Regolith Operations (GMRO) Lab's regolith bin to test the feasibility of 3D additive construction with regolith. The arm has an end effector payload capability of $185 \mathrm{~kg}$ with a maximum reach of $3.14 \mathrm{~m}$. Efforts are underway to deliver: (1) proof-of-concept regolith binding trade study and bench top feasibility testing (2) proof-of-concept regolith and feedstock material storage and transfer mechanism (3) proof-of-concept 3D print head for regolith feedstock (4) proof-of-concept 3D printing (additive regolith construction) structural demonstration.

\section{RESULTS OF KSC DEVELOPMENT WORK}

Previous work in the area of laser processing of non-metallics has focused on high precision micro-structured parts (Balla et al., 2012) or SLS type layered powder bed approaches (Tang et al., 2012). These approaches are appropriate for parts and tooling that require precise geometry and a need to minimize post fabrication machining, however they are not as applicable to the construction of larger structures such has habitats, landing pads, or storage silos. Compared to fabricating small parts, constructing large structures is a different scale that requires a different trade-off between precision, fabrication speed, and energy efficiency. KSC Swamp Works is primarily interested in the larger "construction" end of this scale and has focused on techniques applicable to such. For example, previous work focusing on high precision shapes produced; 
"Dense solid cylindrical parts (f8-10mm, height 25-30mm) without any macroscopic defects ...with $0.25 \mathrm{~mm}$ layer thickness'... at a laser power of $50 \mathrm{~W}$, a scan speed of $20 \mathrm{~mm} / \mathrm{s}$, and a powder feed rate of $12.36 \mathrm{~g} / \mathrm{min}$ ” (Balla, 2012). Typical efficiencies advertised by the Laser Engineering Net Shaping (LENS ${ }^{\mathrm{TM}}$ ) process are $14 \%$, yielding an estimated $1.7 \mathrm{~g} / \mathrm{min}$. fabrication rate. This work identified an appropriate laser energy limit of $2.12 \mathrm{~J} / \mathrm{mm}^{2}(0.53$ $\mathrm{J} / \mathrm{mm}^{3}$ at 0.25 layer thickness) when working on small scale parts (Balla et al., 2012) in order to place a lower limit on melt viscosity. Too low of a viscosity can cause a dripping, running melt which reduces fidelity and precision in the final part. However, working with larger layers and placing less importance on precision, KSC Swamp Work's experiments found that energy levels up to $50 \mathrm{~J} / \mathrm{mm}^{2}$ (11.23 J/mm $\mathrm{mm}^{3}$ at $8.9 \mathrm{~mm}$ layer thickness) could produce cohesive layers with an acceptable amount of viscosity and drip. These results were obtained using a 500W continuous output diode laser at $975 \mathrm{~nm}$ to produce layer thickness' up to $8.9 \mathrm{~mm}(0.35$ ”). A fabrication rate of up to $10 \mathrm{~g} / \mathrm{min}$. was achieved. The higher energy level allowed for faster structure fabrication as described above.

Further, constructing structures from locally available materials requires the process to be robust enough to accept different regolith types with varying mineral contents. Successful fabrication of test materials and bench-top scale freestanding structures was achieved with several types of regolith simulant including:

- $\quad$ Black Point -1 (BP-1), Lunar Regolith Basalt Simulant - NASA KSC

- JSC-1A, Lunar Mare Simulant - NASA Johnson Space Center (JSC)

- NU-LHT-2M, Lunar Highland Type Simulant (2 Medium) - NASA USGS

- Hawaiian Basaltic Tephra from Mauna Kea Volcano, Hai Wahine Valley

- Standard White Construction Sand with 30\% by weight added BP-1

- Cape Canaveral “Jetty Park” Beach Sand with 30\% by weight added BP-1

As the KSC Swamp Work's primary interest lies in the basaltic regolith types and since very little difference was observed in the end product of the different basalt regolith, the majority of the work to date has been conducted with BP-1 as it is extremely economical to obtain. BP-1 has a particle size distribution, that falls within $+/-1$ standard deviation of the lunar regolith average (Rahmatian, 2010, Zeng, 2009). For testing, the BP-1 was not fine sieved, but was passed through a coarse rock screen with $6.3 \mathrm{~mm}(0.25$ ”) spacing, to filter out rocks.

An additional consideration for constructing structures from locally available materials is the water content of the local regolith. This can range to very low, such as lunar regolith, up to potentially 60\%, in some Martian regolith (Horneck, 2008). High water content in the regolith being processed tends to cause voids in the final product. At the minimum $50 \mathrm{~J} / \mathrm{mm}^{2}$ energy level, it was found that the regolith required an additional drying step prior to laser processing to produce reliably void free product. However, at $100 \mathrm{~J} / \mathrm{mm}^{2}$, a separate drying step was not required. It is theorized that the lower viscosity of the melt at higher energy inputs allowed the water vapor to more easily escape the molten regolith before solidifying.

Flexural strength testing (3 point bending) was performed on test coupons of solid processed BP1 and JSC-1A using an Instron load frame testing machine and $100 \mathrm{kN}$ (22,480 lbf) load cell. Multiple samples and tests were conducted for repeatability. Tests results indicated that the samples had strengths better than residential concrete, and similar to some weaker glasses (Bon, 2003). 
Testing of useful shapes beyond test coupons has focused on structures with unsupported spans (which pose a challenge for any Additive Manufacturing process), including cones, hemispherical domes, and ogive domes. Examples are shown below in Figure 2.

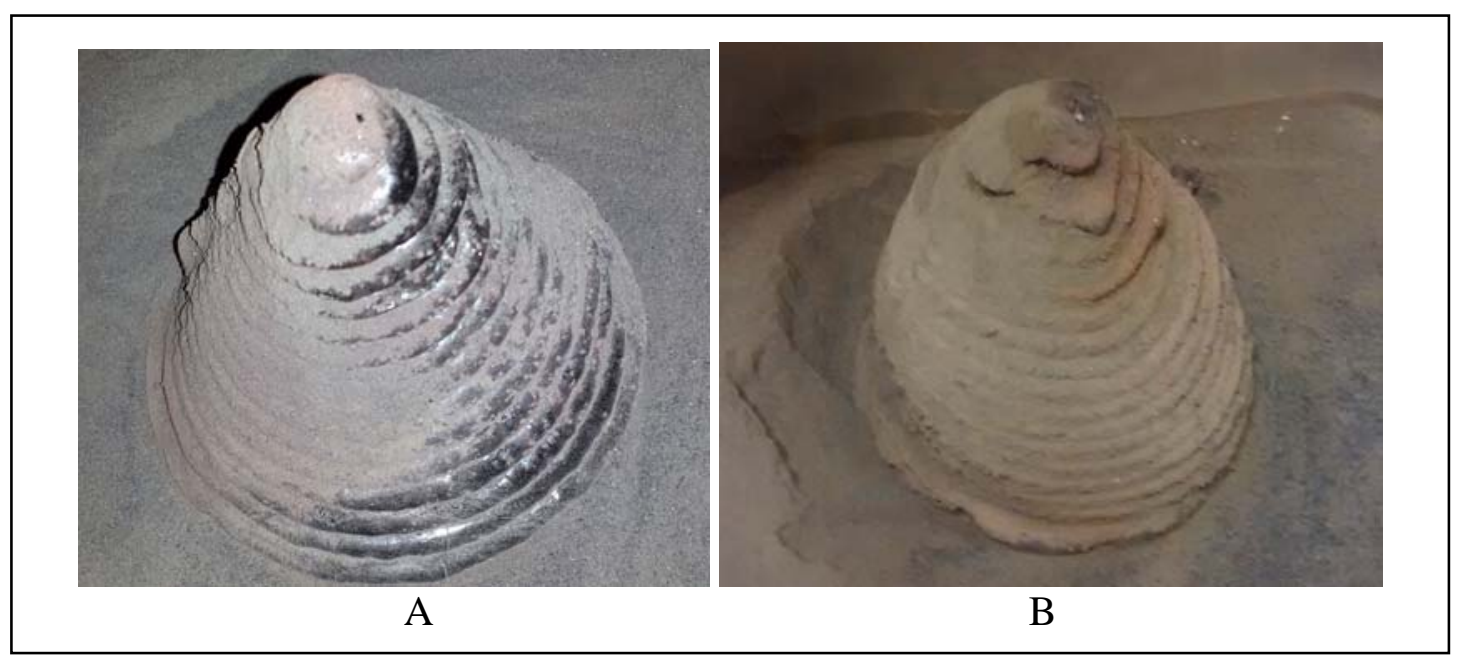

Figure 2. Bench-top scale freestanding structures created by Swamp Works 3D Regolith Construction process: A) BP-1 Hollow Cone Structure; B) BP-1 Hollow Ogive Dome Structure

The results have been promising to date, but only at a bench top scale, however additional work is still ongoing. Future experimental work aims to improve the consistency of material properties throughout the build and scale up the process to create larger structures in the order of several meters in height.

\section{KSC GOALS: 3D ADDITIVE CONSTRUCTION WITH REGOLITH}

The over-arching goals for developing 3D additive construction with regolith, for planetary surface applications are to provide shelter and decrease risk to the crew and mission. Space is a harsh place for humans where they must be protected from the environment. Protection is needed from electromagnetic space radiation, large temperature swings and very steep temperature gradients, micro-meteorite impacts, dust storms, vacuum, fission power plant radiation, rocket blast ejecta at launch/landing, and even moonquakes must be considered. This implies that planetary surface infrastructure will be required whenever space exploration missions will plan to last substantially longer than the current cis-lunar space record of the 3.1 day Apollo 17 lunar surface stay and the 12.6 day round trip to the Moon and back. Infrastructure and civil engineering products are usually heavy since they involve large structures, so it is logical that the heavy concrete-like materials should be substituted with an in-situ regolith-based concrete material. The regolith is available in large quantities on the surface of the Earth's Moon, Mars, Mars moons and some asteroids. The following structures are candidates for 3D Additive Regolith Construction:

- Landing/launch pads

- Blast protection walls/structures

- Consolidated / stabilized slopes for nearby habitat protection to prevent slides 
- $\quad$ Paved access roads

- Hangars for spacecraft and equipment storage

- Sheltered habitats for humans

- Radiation storm shelters at waypoints

- Radiation shielding panels for spacecraft

- Dust free surface stabilized zones (paved areas)

- Thermal inertia pads (wadis) for capturing heat in the day and releasing it during the night with associated shade structures

- Roof structures for sub-surface shelters

- Ablative heat shields for planetary Entry, Descent and Landing (EDL)

- Fission power plant radiation shielding

- Antenna towers for line of sight communication

Additional civil structures will also be needed as mission requirements and architectures evolve.

\section{CONCLUSION}

The technology readiness level (TRL) of this new 3D Additive Construction technology is still low. The authors estimate that its current state is:

"TRL 3 - Analytical and experimental critical function and/or characteristic proof-of concept: Proof of concept validation. Active Research and Development (R\&D) is initiated with analytical and laboratory studies. Demonstration of technical feasibility using breadboard or brass board implementations that are exercised with representative data” [Mankins, 1995].

By performing analysis, testing and lab scale, proof of concept demonstrations, this work has shown that the concept of 3D Additive Construction using basalt fines is feasible, The goal of this experimental investigation was to demonstrate that structures could be built using basalt powder fines, such as the regolith fines that are commonly found on planetary surfaces e.g. Earth's Moon and Mars. Although substantial challenges exist regarding the creation of a material with adequate and repeatable properties for the construction of structures for shelters, production in the vacuum and low gravities of planetary bodies and scaling of the structures to be produced in a larger size, it is a promising start. The problems encountered have been identified and the team at NASA Kennedy Space Center is actively seeking solutions with partners at NASA Marshall Space Flight Center, the University of Southern California and the Pacific International Space Center for Exploration System (PISCES). The goal is to create large structures, of all types, on planetary surfaces, that are suitable for human and robotic equipment shelter.

Since basalt is widely available in any volcanic region on Earth, for example in the volcanic island chain of Hawai'i, near Flagstaff, Arizona in the western United States or near Mt. Vesuvius in Italy, there are also promising terrestrial applications for this technology. The global construction industry has an output valued in the trillions of US dollars per annum, so it is hard to over-emphasize the extreme possibilities of using this new technology to produce economical, energy efficient, rapid, humane and democratic shelter for the Earth's population as well as related industrial applications. Basalt is an environmentally friendly material, and the fines are commonly available at suitable quarries, as a waste material from gravel crushing operations. 
The report “Global Construction 2020”, projects that: “Over the next 7 years it has been estimated that the global construction industry will grow from US \$7.2 trillion to over US \$12 trillion. A total of $\$ 97.7$ trillion will be spent on construction globally over the next ten years. The new 2025 report forecasts the volume of construction output will grow by more than $70 \%$ to $\$ 15$ trillion worldwide by 2025 . The global construction industry currently represents about $13 \%$ of global Gross Domestic Product (GDP) and this number will increase to 15\% in 2020. Countries poised to undergo the largest growth include China, India, Russia, Brazil, Poland and the US”, (G. C., \& Economics, O. 2013). This represents a substantial economic opportunity in domestic and export markets for the US (or other nationality) pioneers that develop this 3D Additive Construction technology. The technical results of this terrestrial activity and investments can then be applied to expand our economic sphere of influence beyond the Earth and into the solar system, where the bulk of our accessible resources reside. Ultimately, the human condition could improve through utilization of essentially unlimited solar energy and very large quantities of space resources.

\section{REFERENCES}

Balla, V. K., Roberson, L. B., O'Connor, G. W., Trigwell, S., Bose, S., and Bandyopadhyay, A. (2012) "First demonstration on direct laser fabrication of lunar regolith parts." Rapid Prototyping J., 18 (6), 451-457.

Bon, E. (2003). "Structural Opportunities for Glass," Master's Thesis, Massachusetts Institute of Technology.

G. C., \& Economics, O. (2013). Perspectives, Global Construction 2020. GCPOE, London.

Gosau, J. M. (2012) "Regolith Stabilization and Building Materials for the Lunar Surface.” ASCE Aerospace Division International Conference on Engineering, Science, Construction, and Operations in Challenging Environments (Earth \& Space 2012). Pasadena, California, USA, 15 18 Apr 2012. Reston, Virginia, USA: American Society of Civil Engineers.

Horneck, G. (2008). "The microbial case for Mars and its implications for human expeditions to Mars". Acta Astronautica 63 (7-10): 1015

Howe, A. S., Wilcox, B., McQuin, C., Townsend, J., Rieber, R., Barmatz, M., and Leichty, J. (2013). "Faxing Structures to the Moon: Freeform Additive Construction System (FACS)" AIAA 2013-5437, AIAA SPACE 2013 Conference and Exposition, September 10-12, 2013, San Diego, CA.

Khoshnevis, B., Thangavelu, M., Yuan, X., and Zhang, J. (2013). “Advances in Contour Crafting Technology for Extraterrestrial Settlement Infrastructure Buildup.” AIAA 2013-5438, AIAA SPACE 2013 Conference and Exposition, September 10-12, 2013, San Diego, CA.

Mankins, J. C. (1995). Technology readiness levels. NASA White Paper, April, 6, 1995.

Mearian, L. (2014). “3D printer constructs 10 buildings in one day from recycled materials.” Computer World, July 2, 2014.

Tang, Y., Fuh, J.Y.H., Loh, H.T., Wong, Y.S., and Lu, L. (2003) "Direct laser sintering of a silica sand.” Materials and Design, 24 (2003), 623-629.

Rahmatian, L. A., \& Metzger, P. T. (2010). Soil Test Apparatus for Lunar Surfaces. American Society of Civil Engineers, Earth and Space.Conference, Honolulu, Hawaii.

Zeng, Xiangwu, Chunmei He, and Heather Oravec, (2009). "Geotechnical Properties of JSC-1A Lunar Soil Simulant.” J. Aerospace Eng., ASCE Aerospace Division. 